\title{
INFLUENCE DE L'APPROVISIONNEMENT EN EAU SUR LA STRUCTURE INTERNE DES ACCROISSEMENTS ANNUELS
}

\author{
EXPÉRIENCE D'IRRIGATION SUR PINS SYLVESTRES
}

H. POLGE et R. KELLER

Station de Recherches sur la Qualité des Bois, Centre national de Recherches forestières, 54 - Nancy

Institut national de la Recherche agronomique

\section{SOMMAIRE}

Une comparaison est faite entre 16 arbres traités et 16 témoins pour étudier les conséquences sur la largeur et les composantes de la densité du cerne 1967 d'un apport d'eau équivalent à $70 \mathrm{~mm}$ de pluie effectué au début d'août 1967 sur des Pins sylvestres de 28 ans.

\section{1. - BUT DE L'EXPÉRIENCE}

Les variations de structure anatomique du bois à l'intérieur des cernes successifs sont, on le sait, sous la dépendance d'un grand nombre de facteurs : hérédité, sol, climat, traitement, etc. qui interfèrent les uns aux autres, en sorte que le déterminisme de critères aussi simples que le pourcentage de bois final, ou les densités propres du bois de printemps et du bois d'été par exemple, est encore très peu connu.

Le but, de l'expérience très sommaire dont il est rendu compte ici, a été d'étudier l'influence d'un facteur simple, l'approvisionnement en eau, sur les principales caractéristiques d'un accroissement annuel telles qu'elles peuvent être mesurées sur les courbes de variations de la densité du bois.

La situation paraissait assez favorable pour entreprendre une telle étude en 1967 par suite de la sécheresse relative qui avait sévi dans la région nancéienne durant les premiers mois de l'année, et qui s'était aggravée en juin, puis en juillet.

La figure 1 représente, sous forme de graphique, les précipitations cumulées des 7 premiers mois, relevées au poste météorologique de l'Arboretum d'Amance 


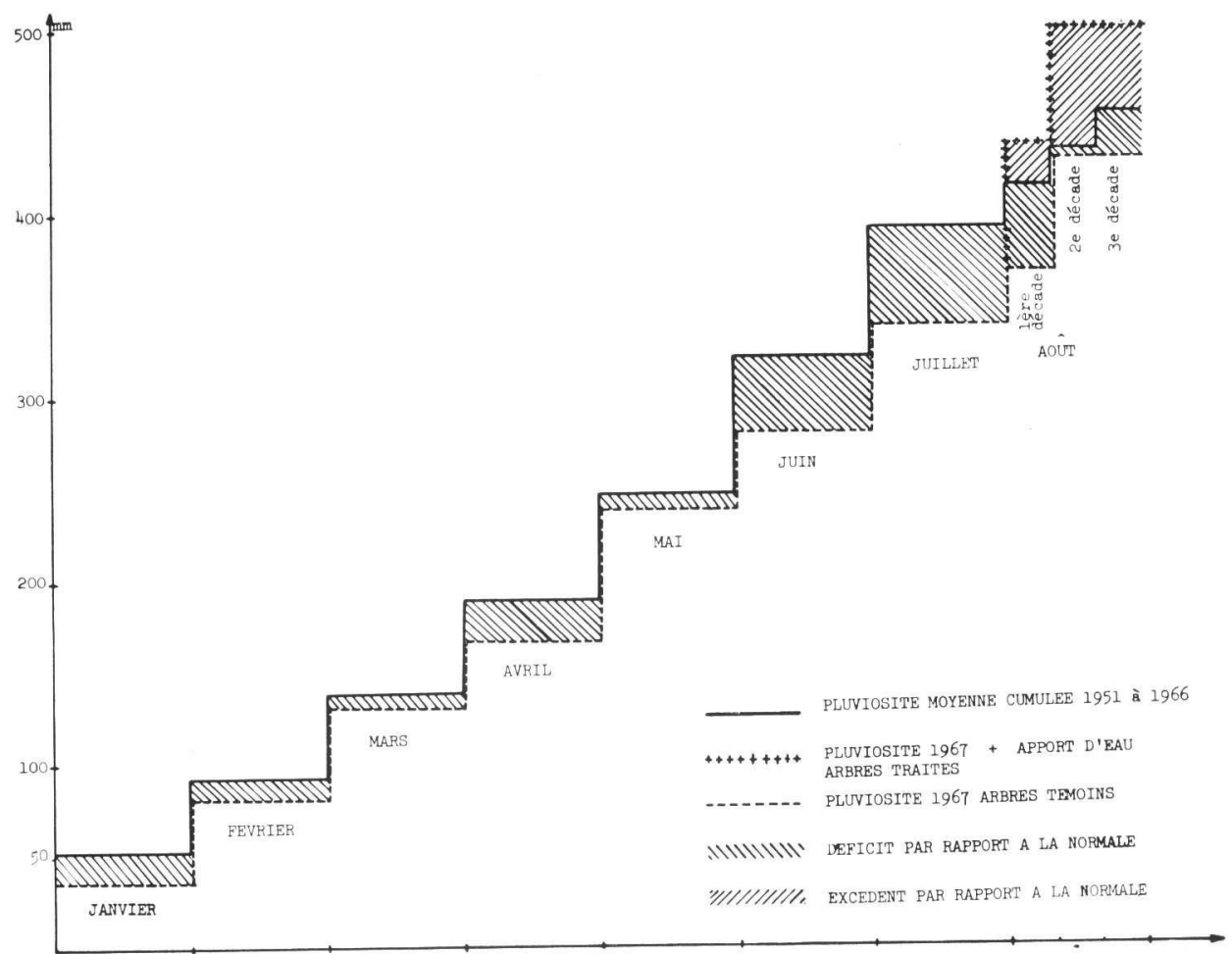

Fig. 1. - Précipitations cumulées des 7 premiers mois et pluviosité moyenne cumulée des 15 années précédentes (postes météorologiques de l'Arboretum d'Amance)

où a été entreprise cette expérience, avec, à titre de comparaison, la pluviosité moyenne cumulée des 15 années précédentes. En outre, pour le mois d'août, durant lequel devait avoir lieu l'intervention projetée, les mêmes renseignements sont donnés séparément pour chacune des deux premières décades, et pour les 11 derniers jours du mois.

\section{2. - MATÉRIEL ET MÉTHODE}

L'expérience a été assise sur 32 arbres de 28 ans pris en nombre égal parmi les dominants et parmi les dominés du placeau 54 de l'expérience comparative de provenances de Pins sylvestres d'Amance, et choisis de façon telle qu'ils présentent deux à deux des caractéristiques aussi voisines que possible au point de vue diamètre de la tige et ampleur du houppier, tout en étant suffisamment distants les uns des autres pour que leurs systèmes radiculaires puissent être considérés comme indépendants.

Un arbre de chacune des paires ainsi constituées a été désigné comme témoin, et le second a reçu, le 3 ou le 4 août, un apport d'eau de 5001 ; pour chaque individu traité, cette irrigation a été réalisée sous forme d'un déversement unique fait à la tonne d'arrosage à l'intérieur d'un petit bassin de 1,50 m de rayon délimitée par un simple bourrelet de terre rapportée.

L'eau supplémentaire ainsi fournie représente sur la surface considérée une lame de $70 \mathrm{~mm}$ d'épaisseur, mais cette équivalence n'est qu'assez grossière car, contrairement à ce qui se passe 
pour les précipitations atmosphériques, il n'y a pas eu interception par les houppiers ; en contrepartie, on aurait pu craindre, en raison de la grande aridité du sol au moment de l'expérience, que le déversement, effectué sur une surface unitaire d'environ $7 \mathrm{~m}^{2}$, ne bénéficie en fait à une zone beaucoup plus étendue ; on a vérifié, en procédant à un sondage à proximité immédiate de l'un des bassins, qu'il n'en était rien, et que la terre y était restée parfaitement sèche.

Par ailleurs, et pour faciliter la réalisation matérielle de l'expérience, la couverture vivante, au reste très peu abondante, a été enlevée au pied des arbres à irriguer, sans que la même opération soit faite pour les témoins ; nous ne pensons pas que cette différence de traitement ait pu constituer une source d'hétérogénéité supplémentaire à l'avantage des premiers, car il n'y a pas eu de véritable travail du sol, et il est même possible que ce léger sarclage ait, au contraire, facilité à leur détriment l'évaporation d'une partie de l'eau reçue ultérieurement.

Sous ces réserves, on peut observer sur la figure 1 que le déficit de pluviosité par rapport à la moyenne des 15 années précédentes s'est transformé, au mois d'août, en excédent pour les arbres traités, ce qui n'a pas été le cas pour les témoins.

Cependant, des pluies importantes se sont produites ce même mois $(30 \mathrm{~mm}$ durant la première décade et 61 durant la deuxième), en sorte que les écarts entre témoins et arbres traités n'ont pu que se trouver considérablement réduits par rapport à ceux que l'on aurait pu provoquer si la sécheresse avait persisté durant le mois où l'intervention a eu lieu.

Les échantillons destinés à étudier les effets de l'apport d'eau effectué ont consisté en carottes de sondage prélevées à 1,30 $\mathrm{m}$ de hauteur sur la face Nord de tous les arbres en cause.

Les courbes de variations de la densité ont été obtenues par analyse densitométrique de clichés radiographiques suivant une méthode déjà décrite (Polge H., 1966).

Tous les échantillons ont été radiographiés ensemble sur un même film, et un rapport d'agrandissement de 20 a été utilisé pour tous les profils densitométriques qui en ont été tirés ; les chiffres donnés ci-dessous pour les diverses largeurs sont donc à diviser par 20 si l'on veut obtenir les valeurs moyennes exprimées en mm.

$$
\text { 3. - RÉSULTATS }
$$

\section{1. - Contrôle de la similitude des arbres traités et des témoins avant l'expérience}

Le cerne 1966 a été analysé en détail afin de s'assurer qu'il n'existait, entre les divers arbres à étudier, aucune différence initiale significative.

\section{TABLEAU 1}

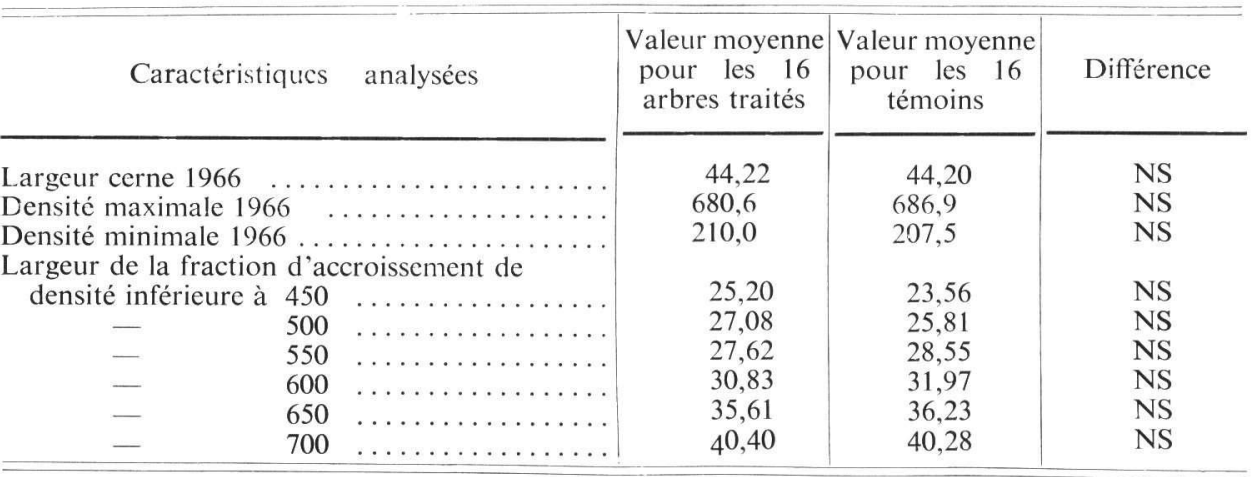


On voit qu'aucune différence significative n'existe entre témoins et arbres traités pour les 9 caractéristiques analysées ; les écarts sont extrêmement faibles pour les largeurs de cernes et les densités maximales ou minimales annuelles; ils sont un peu plus importants en valeur relative pour les fractions de l'accroissement annuel 1966 de densité inférieure aux diverses densités de référence, mais ces différences ne sont pas toujours de même signe et ne paraissent dues qu'au nombre relativement peu élevé d'échantillons étudiés.

\section{2. - Effets du traitement}

Pour tenir compte des faibles écarts (non significatifs on l'a vu) relevés ci-dessus, on a analysé, pour étudier l'effet de l'apport supplémentaire d'eau réalisé, non pas le cerne 1967, mais la différence en valeur algébrique entre le cerne 1966 et le cerne 1967.

En outre, l'expérience a porté sur un trop petit nombre d'arbres pour permettre de mettre en évidence l'influence éventuelle de l'étage sociologique qu'ils occupent dans le peuplement; on peut seulement noter que les dominés semblent avoir été plus sensibles au traitement que les dominants, mais la différence n'est pas significative, et les deux catégories en cause ont par suite été regroupées dans l'analyse statistique d'ensemble dont les résultats sont donnés dans le tableau ci-dessous :

TABLEAU 2

\begin{tabular}{|c|c|c|c|c|}
\hline Caractéristiques & analysées & $\begin{array}{l}\text { Valeur moyenne } \\
\text { pour les } 16 \\
\text { arbres traités }\end{array}$ & $\begin{array}{l}\text { Valeur moyenne } \\
\text { pour les } 16 \\
\text { témoins }\end{array}$ & Test «t» \\
\hline $\begin{array}{cc}\text { Différence 1966-1967 pour } 1 \\
\text { - largeur totale du cerne } \\
\text { - largeur de la fraction } \\
\text { densité inférieure à } 450 \\
- & 500 \\
- & 550 \\
- & 600 \\
\text { - } & 650 \\
\text { - largeur de la fraction } \\
\text { densité supérieure à } 450 \\
\text { - } & 500 \\
\text { - } & 550 \\
\text { - } & 600 \\
\text { - } & 650 \\
\text { densité maximale } & 700 \\
\text { - densité minimale } & \ldots\end{array}$ & 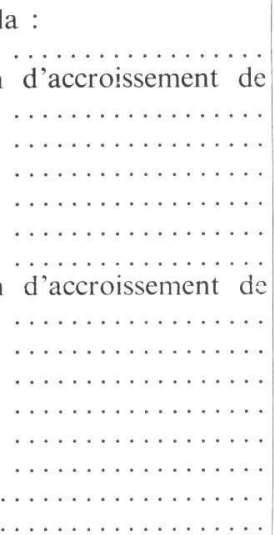 & $\begin{array}{l}-4,67 \\
-\quad 1,03 \\
-0,20 \\
-1,45 \\
-2,69 \\
-3,60 \\
-\quad 4,84 \\
-3,64 \\
-4,47 \\
-\quad 3,22 \\
-1,98 \\
-1,07 \\
+0,17 \\
-21,6 \\
-31,0\end{array}$ & $\begin{array}{l}+5,12 \\
+\quad 5,19 \\
+5,75 \\
+6,56 \\
+7,87 \\
+5,19 \\
+4,94 \\
-0,07 \\
-0,63 \\
-1,43 \\
-2,65 \\
-0,07 \\
+0,19 \\
-24,1 \\
-41,9\end{array}$ & $\begin{array}{c}-2,71^{* *} \\
-2,92^{* *} \\
-2,33^{*} \\
-2,65^{* *} \\
-3,13^{* *} \\
-2,47^{*} \\
-2,68^{* *} \\
\text { NS } \\
\text { NS } \\
\text { NS } \\
\text { NS } \\
\text { NS } \\
\text { NS } \\
\text { NS } \\
\text { NS }\end{array}$ \\
\hline
\end{tabular}

On peut faire les constatations suivantes :

Malgré 1'importance relativement réduite de l'apport d'eau effectué, et malgré les précipitations des deux premières décades du mois d'août qui ont, à très peu 
près, comblé le déficit de pluviosité du début de l'année 1967, le traitement a entraîné des différences significatives, ou très significatives, sur un assez grand nombre de caractéristiques ; le cerne 1967 est en particulier nettement plus large que le cerne 1966 pour les arbres irrigués, alors qu'il est au contraire plus étroit pour les témoins (le gain moyen est de $0,45 \mathrm{~mm}$, soit en valeur relative $23 \%$ ), et il en est de même pour les fractions de largeur de cernes de densité inférieure à toutes les valeurs de référence retenues.

Le fait que l'on enregistre des écarts importants, aussi bien dans les accroissements annuels totaux que dans la partie à faible densité de ceux-ci, nous a amenés à tester également les largeurs de cernes de densité supérieure aux mêmes densités de base, dont on ne pouvait, a priori, savoir si elles seraient, elles aussi, affectées par le traitement ; on voit qu'il n'en est rien, puisque les différences 1966-1967 ne sont, en ce qui les concerne, pas significatives.

On peut en conclure, ce que l'on ignorait également au départ, que l'irrigation artificielle à laquelle on a procédé est intervenue durant la phase de croissance correspondant à la formation du bois de printemps ; cet apport d'eau a entraîné une augmentation significative de la largeur de ce dernier, et n'a eu pratiquement aucune répercussion sur la fraction dense de l'accroissement, pas plus que sur les densités maximales et minimales annuelles, et tout se passe comme si le traitement appliqué n'avait fait que différer dans le temps les divers phénomènes physiologiques responsables de la formation du bois d'été, sans en altérer ni l'ampleur ni le rythme.

\section{3. - Comparaison globale entre les années 1966 et 1967}

On a vu qu'il n'y avait pas de différence significative entre arbres traités et témoins sur les différences entre les densités maximales ou les densités minimales de 1966 et 1967 ; ceci nous a incité à regrouper les résultats pour l'ensemble des 32 arbres du dispositif afin de voir si les variations des conditions météorologiques avaient exercé une influence sur ces deux caractéristiques; les résultats de cette comparaison sont consignés dans le tableau ci-dessous :

TABLEAU 3

\begin{tabular}{l|l|l|l}
\hline & 1966 & 1967 & Test « $t$ » \\
& & & \\
Densité maximale $\ldots \ldots \ldots \ldots \ldots \ldots \ldots \ldots \ldots \ldots$ & 688,7 & 711,7 & $-3,62^{* *}$ \\
Densité minimale $\ldots \ldots \ldots \ldots \ldots \ldots \ldots \ldots \ldots \ldots$ & 208,7 & 245,3 & $-2,71^{* *}$ \\
\hline
\end{tabular}

L'augmentation de la densité maximale de 1966 à 1967 et celle de la densité minimale sont donc significatives au seuil de 1/100.

On a vu dans une étude précédente (Polge H., 1966, op. cit.) que la densité maximale était en corrélation étroite :

- pour le Douglas, avec la pluviosité cumulée des mois d'août, septembre et octobre (coefficient de corrélation de $-0,89$ avec 10 degrés de liberté), 
- pour le Grandis, avec celle des mois d'août et septembre seulement (coefficient de corrélation de $-0,77)$.

Quant à la densité minimale, elle était fortement liée à la pluviosité de mars, avril et mai pour le Grandis $(r=-0,78)$, cependant que celle du Douglas paraissait beaucoup plus difficile à rattacher aux diverses précipitations mensuelles.

Or, si l'on met en parallèles les quantités d'eau reçues en 1966 et 1967 à l'Arboretum d'Amance, on obtient les résultats suivants :

TABLEAU 4

\begin{tabular}{|c|c|c|c|c|c|c|c|c|c|c|c|}
\hline & & Janv. & Fév. & Mars & Avril & Mai & Juin & Juil. & Août & Sept. & Oct. \\
\hline Pluviosité & 66 & 61,2 & 52,8 & 36,1 & 87,0 & 55,3 & 57,7 & 52,0 & 91,2 & 41,4 & 75,7 \\
\hline mensuelle & 67 & 37,4 & 43,9 & 59,2 & 36,5 & 71,9 & 41,8 & 57,8 & 91,5 & 123,4 & 89,3 \\
\hline Pluviosité & 66 & 61,2 & 114,0 & 150,1 & 237,1 & 292.4 & 350,1 & 402.1 & 493,3 & 534,7 & 610,4 \\
\hline cumulée & 67 & 37,4 & 81,3 & 130,5 & 167,0 & 238,9 & 280,7 & 338,5 & 430,0 & 553,4 & 642,7 \\
\hline
\end{tabular}

La comparaison ci-dessus laisse à penser qu'il y a chez le Pin sylvestre également une tendance générale à une augmentation des densités maximales et minimales annuelles lorsque la pluviosité diminue, mais le phénomène paraît ne pas suivre exactement les mêmes lois que pour les deux essences exotiques auxquelles il est fait allusion ci-dessus ; on peut observer en particulier que la pluviosité cumulée des mois d'août et septembre 1967 est supérieure de plus de $80 \mathrm{~mm}$ à celle des mois correspondants de l'année précédente (l'écart atteint même $100 \mathrm{~mm}$ pour l'ensemble des trois mois : août, septembre et octobre), et cependant, on l'a vu, la densité maximale a été, de façon très significative, plus élevée pour la deuxième année que pour la première.

Il semblerait donc que, pour le Pin sylvestre, comme pour le Grandis ou le Douglas, les précipitations des premiers mois de l'année exercent une influence directe sur la valeur de la densité minimale annuelle ; mais, pour la densité maximale, un déficit global élevé se produisant au début de la saison de végétation (il dépassait, dans le cas particulier, $60 \mathrm{~mm}$ à la fin du mois de juillet) entraînerait une augmentation de la densité, quelle que soit l'importance de la nappe d'eau reçue ultérieurement.

\section{4. - DISCUSSION ET CONCLUSION}

Les résultats de cette expérience recoupent assez bien ceux d'études beaucoup plus poussées entreprises aux Etats-Unis sur le même sujet.

ZAHNER et al. (1964) ont analysé les caractéristiques anatomiques de Pinus resinosa de 20 ans, les uns irrigués de façon à maintenir le sol à la capacité au champ, les autres soumis à partir de la mi-juin à une sécheresse à peu près totale par creusement autour de leurs racines d'une tranchée destinée à les isoler du reste du peuplement, et par pose, sur la zone ainsi délimitée, d'une toile imperméable. 
Ils ont montré que les arbres arrosés différaient de ceux qui avaient été soumis à une aridité artificielle surtout par un plus grand développement de leur bois initial (130 cellules en moyenne dans le sens radial contre 80 ), alors que les observations faites sur le bois final étaient contradictoires et les écarts les concernant beaucoup plus faibles ; le nombre moyen de cellules de bois d'été était en effet plus élevé dans les arbres irrigués si l'on se réfère à la définition de Mork (30 contre 19), mais par contre, plus réduit lorsqu'on ne prend en considération que les cellules nettement aplaties dans le sens radial (5 contre 10).

Quant aux causes des modifications anatomiques qui interviennent en fonction de l'approvisionnement en eau, elles ont été étudiées en détail par LARSON (1963) travaillant également sur des Pinus resinosa, mais à l'état de semis de 5 ans; il a montré qu'une sécheresse artificielle de courte durée intervenant pendant la période de formation du bois de printemps entraînait une diminution de la longueur des aiguilles, une réduction corrélative de la teneur en auxine (mise en évidence par des tests avoine), et finalement la production d'un faux cerne ; si l'approvisionnement en eau redevient normal par la suite, les plants en cause recommencent donc à produire du bois de printemps ; mais si l'élaboration de l'auxine est freinée au même moment, soit par défoliaison, soit par passage artificiel en jours courts, le retour à des trachéides de gros diamètre et à parois minces ne se produit plus, et le passage au bois d'été devient irréversible.

L'auteur en déduit que la sécheresse agit de façon directe sur la croissance du méristème terminal, mais que son action sur les dimensions des trachéides ne s'exerce, au contraire, qu'indirectement par l'intermédiaire d'une réduction de l'apport en auxine.

Selon l'hypothèse émise, les substances de croissance interviendraient essentiellement pour augmenter la plasticité de la membrane primaire, plasticité à défaut de laquelle une augmentation de la pression de sève serait incapable d'entraîner un agrandissement concomitant du diamètre des cavités cellulaires.

Quoi qu'il en soit, la petite expérience dont il est rendu compte ci-dessus ne constitue pour nous qu'un premier essai dont les résultats, assez encourageants, demandent à être confirmés, à la faveur de nouvelles circonstances météorologiques favorables, en traitant un plus grand nombre d'individus d'essences variées, et en essayant d'intervenir pendant la période de production du bois final, ce qui compléterait utilement les informations recueillies à la suite du présent travail.

Au reste, il est clair, et l'analyse de LARSON le prouve, que l'on ne progressera sérieusement dans la connaissance des phénomènes fondamentaux liant les variations des divers facteurs de la production forestière à celles de la structure interne des accroissements annuels que si l'on peut mettre en évidence les causes des transformations observées.

Des études biochimiques poussées devront donc être entreprises le plus tôt possible à cette fin, en raison du rôle primordial que paraissent jouer en la matière les hormones de croissance et les substances antagonistes. 


\section{REMERCIEMENTS}

Nous sommes redevables des renseignements météorologiques, concernant l'Arboretum d'Amance, à la Station de Sylviculture et de Production du C.N.R.F., cependant que la Station d'Amélioration des Arbres forestiers a mis à notre disposition le peuplement de Pins sylvestres intéressé, et nous a fourni les moyens matériels nécessaires pour mener à bien cette expérience. Que l'une et l'autre soient ici remerciées pour l'aide efficace qu'elles nous ont apportée.

\section{SUMMARY}

INFLUENCE OF WATER SUPPLY ON THE INTERNAL STRUCTURE OF ANNUAL RINGS. LITTLE IRRIGATION EXPERIMENT ON SCOTCH PINES

After a dry weather during the beginning of the year (rain deficit of $54 \mathrm{~mm}$ for the first seven months), an irrigation trial has been undertaken on the 3rd and 4th of August 1967 with 18 years old Scotch Pines ; 5001 of water, equivalent to a rain of $70 \mathrm{~mm}$, have been discharged at once within little basins made $1,5 \mathrm{~m}$ all around each tree.

The characteristics of the ring 1967 have been studied by densitometric analysis of radiographs.

Table 1 shows that the 16 treated trees and the 16 control trees were quite similar before the experiment.

Because of the irrigation, have significantly increased the ring width and the portions of the annual ring having a density lower than several given densities ranging from 450 to $700 \mathrm{~g} / \mathrm{dm}^{3} ;$ but all the dense part of the ring has not been modified, and this irrigation only seems to have caused a translation of the latewood without changing its essential characteristics: width and instantaneous densities (table 2).

A comparison between the rings 1966 and 1967 of all the 32 trees of the experiment leaves to think that insufficient rains during the first months of the year, bring about an increase of the minimum annual density; but for the Scotch Pine an important water deficit occuring before the beginning of the latewood production also leads to an heavier maximum annual density, even if this dry weather is followed by copious rains before the cessation of the cambial activity (tables 3 and 4 ).

\section{ZUSAMMENFASSUNG}

Einfluss der Wasserversorgung auf die interne Struktur der Jahrringe EIN KLeINer IRrigationsversuch VON Pinus sylvestris $L$.

Die ersten sieben Monate des Jahres 1967 erbrachten ein Wasserdefizit in der Gesamthöhe von $54 \mathrm{~mm}$. Am 3. und 4. August 1967 wurde daher ein Irrigationsversuch an 18 jährigen Weisskiefern durchgeführt, wobei eine einmalige Wassergabe von 5001 pro Baum - dies entsprach einer Niederschlagsmenge von $70 \mathrm{~mm}$ - in einen kreisrunden, den Baum umgebenden Behälter (Durchmesser : $300 \mathrm{~cm}$ ) eingebracht wurde.

Die wesentlichen Merkmale des Jahrringes 1967 wurden durch eine Mikrodensitometeranalyse von Röntgenaufnahmen festgestellt.

Die Tabelle 1 zeigt, dass die 16 behandelten und die 16 unbehandelten Bäume zu Versuchsbeginn untereinander völlig vergleichbar waren.

Die Irrigation erbrachte eine signifikative Erhöhung der Jahrringbreiten und die Anteile des Jahrreszuwachses mit einer geringeren Dichte als verschiedene Bezugswerte haben eine Dichte in den Grenzen von 450 bis $700 \mathrm{~g} / \mathrm{dm}^{3}$. Im Gegensatz dazu wurden jedoch die dichteren Teile des Jahrringes nicht verändert und es scheint, dass die Wassergabe nur eine zeitliche Verschiebung der Spätholzbildung verursacht ohne die wesentlichen Merkmale (Spätholzbreite und die entsprechenden verschiedenen Dichtewerte) zu beeinflussen. (Tab. 2).

Ein Vergleich der Jahrringe 1966 und 1967, der das gesamte Versuchskollektiv von 32 Bäumen betraf, lässt vermuten, dass eine ungenügende Niederschlagsmenge während der ersten Monate des Jahres eine Erhöhung der Minimaldichte verursacht und ein bedeutendes Wasserdefizit vor Beginn der Spätholzbildung gleichfalls eine Erhöhung der Maximaldichte hervorruft, auch dann, wenn sich ein Wasserüberschuss vor Einstellung des Kambialwachstums ergibt. (Tab. 3 und 4). 


\section{RÉFÉRENCES BIBLIOGRAPHIQUES}

Larson Ph.R., 1963. The indirect effect of drought on tracheid diameter in Red pine. Forest Sci., 9, 1, 52-62.

Polge H., 1966. Etablissement des courbes de variation de la densité du bois par exploration densitométrique de radiographies d'échantillons prélevés à la tarière sur des arbres vivants. Applications dans les domaines technologique et physiologique. Thèse Docteur ès sciences appliquées. Ann. Sci. forest., 23, 1.

Zahner R., Lotan J.E., Baughman W.D., 1964. Earlywood-latewood features of red pine grown under simulated drought and irrigation. Forest Sci., 10, 3, 361-370. 